\title{
A Search for Neutrinos from Decaying Dark Matter in Galaxy Clusters and Galaxies with IceCube
}

\section{The IceCube Collaboration}

(a complete list of authors can be found at the end of the proceedings)

E-mail: minjin.jeong@g.skku.edu

The observed dark matter abundance in the Universe can be explained with non-thermal, heavy dark matter models. In order for dark matter to still be present today, its lifetime has to far exceed the age of the Universe. In these scenarios, dark matter decay can produce highly energetic neutrinos, along with other Standard Model particles. To date, the IceCube Neutrino Observatory is the world's largest neutrino telescope, located at the geographic South Pole. In 2013, the IceCube collaboration reported the first observation of high-energy astrophysical neutrinos. Since then, IceCube has collected a large amount of astrophysical neutrino data with energies up to tens of PeV, allowing us to probe the heavy dark matter models using neutrinos. We search the IceCube data for neutrinos from decaying dark matter in galaxy clusters and galaxies. The targeted dark matter masses range from $10 \mathrm{TeV}$ to $10 \mathrm{PeV}$. In this contribution, we present the method and sensitivities of the analysis.

Corresponding authors: M. Jeong ${ }^{1 *}$

${ }^{1}$ Sungkyunkwan University

* Presenter

$37^{\text {th }}$ International Cosmic Ray Conference (ICRC 2021)

July 12th - 23rd, 2021

Online - Berlin, Germany 


\section{Introduction}

The existence of dark matter has been well established by a variety of astronomical observations, but the particle nature of dark matter still remains unknown. Various theories, beyond the Standard Model of particle physics, propose candidates for dark matter. Among those, Weakly Interacting Massive Particles (WIMPs) have been the most extensively studied candidates, for a number of reasons. WIMPs naturally explain the abundance of dark matter in the present Universe [1]. On the other hand, the mass scale allowed for WIMPs is limited up to $\sim 100 \mathrm{TeV}$, due to the unitarity bound [2], and thus accessible by current instruments. However, decaying heavy dark matter, which was non-thermally produced in the early Universe and has a longer lifetime than the age of the Universe, is also a viable candidate for dark matter [3, 4].

Neutrinos are useful tools for testing dark matter hypotheses. When dark matter pair-annihilates or decays, neutrinos could be produced directly or through the decay of the primary products. Hence, the properties of dark matter can be constrained by measuring the neutrino flux from celestial objects which host a large amount of dark matter. Neutrinos have several advantages over other messenger particles. They are not affected by magnetic fields and have extremely small cross-sections. Thus, their directions can be better associated with their origins. Also, they are barely absorbed at their production site. This provides unique opportunities to search for dark matter in the core of the Sun and the Earth [5-7].

The IceCube Neutrino Observatory [8] is a cubic-kilometer scale neutrino telescope deployed in the deep glacial ice in the Antarctica. The IceCube collaboration has reported observations of high-energy astrophysical neutrino events [9-11]. Theoretical studies suggest that some of these astrophysical neutrinos could originate from the decay of dark matter particles [12-15]. Recent IceCube analyses looked for neutrinos from dark matter decay in the Galactic Halo and isotropic neutrinos from extragalactic dark matter decay $[16,17]$. These searches provide highly competitive limits on the dark matter decay lifetime, demonstrating that IceCube is sensitive to probe decaying heavy dark matter hypotheses.

In this contribution, we present a search for neutrinos from the decay of dark matter particles in galaxy clusters and galaxies, which utilizes track-like events observed in IceCube. In section 2, we describe the expected signal and background of this analysis. In section 3, we discuss the data sample and statistical methods used in this work. Then we present the sensitivities of this analysis in section 4 and conclude in section 5 .

\section{Signal and Background}

The differential neutrino flux expected from the decay of dark matter particles in an extragalactic source is calculated as the following:

$$
\frac{d \Phi_{v}}{d E_{v}}\left(E_{v}\right)=\frac{1}{4 \pi m_{\chi} \tau_{\chi}} \frac{d N_{v}}{d E_{v}}\left(E_{v}\right) \int_{0}^{\Delta \Omega} d \Omega \int_{0}^{\infty} \rho_{\chi}(l \hat{n}) d l,
$$

with $m_{\chi}$ being the dark matter mass, $\tau_{\chi}$ the dark matter lifetime, $\hat{n}$ the direction of the line-of-sight, and $d N_{v} / d E_{v}$ the differential neutrino spectrum per dark matter particle decay. We assume that dark matter decays into a pair of Standard Model particles with a branching ratio of $100 \%$ and 
calculate the energy spectrum of neutrinos in the final state. This calculation is done for different dark matter masses and decay channels using the $\chi$ arov software package [18]. This package takes into account the electroweak corrections and the neutrino oscillation from the source to the Earth. Figure 1 shows the expected spectra of muon neutrinos (and anti-muon neutrinos) at the Earth, for dark matter masses of $10 \mathrm{TeV}$ and $10 \mathrm{PeV}$, decaying through different dark matter channels. The neutrino oscillation inside the Earth is negligible, as we use neutrinos with energies higher than $100 \mathrm{GeV}$. The attenuation of high-energy neutrino fluxes due to absorption in the Earth is accounted for using the Nugen software packages [19].

The spatial distribution of the neutrino flux depends on the distribution of dark matter in the source. In this analysis, we use galaxy clusters, the Andromeda galaxy (M31), and satellite dwarf galaxies of the Milky Way as targets. For dwarf galaxies, we adopt the dark matter halo models presented in [20]. The authors use the Zhao density profile [21] to describe the dark matter halos in the dwarf galaxies. For galaxy clusters, we adopt the models presented in [22]. Here, the dark matter halos of these clusters are described using the Navarro-Frenk-White (NFW) profile [23] which is a special case of the Zhao profile. The model for the dark matter distribution in the Andromeda galaxy is adopted from [24] and is based on the Einasto profile [25]. Lastly, we assume that the dark matter halos of the sources do not contain subhalos, as the signal from decaying dark matter is not highly sensitive to subhalos.

Given the dark matter halo models for different sources, we evaluate the integral in Eq. 1, conventionally referred to as the D-factor, for each of the sources with $\Delta \Omega=2 \pi \cos \theta$. Here, $\theta$ denotes the angular distance from the center of the source. Then, we select the targets with larger D-factors than $10^{18} \mathrm{GeV} / \mathrm{cm}^{2}$, which are listed in Table 1. $\theta_{\text {max }}$ denotes the angular distance from the source at which the D-factor reaches its maximum, while $D_{\max }$ is the D-factor calculated up to $\theta=\theta_{\max }$. We use the CLUMPY software package $[26,27]$ to calculate $D_{\max }$ and the spatial distribution of the neutrino flux from the targets. The locations of the selected targets in the sky are depicted in Figure 2.

The expected background includes the neutrinos produced by cosmic-ray interactions with Earth's atmosphere and isotropic astrophysical neutrinos. The background is expected to be uniform in right ascension, as the detector rotates along the Earth's rotation axis. Thus, the scrambled data
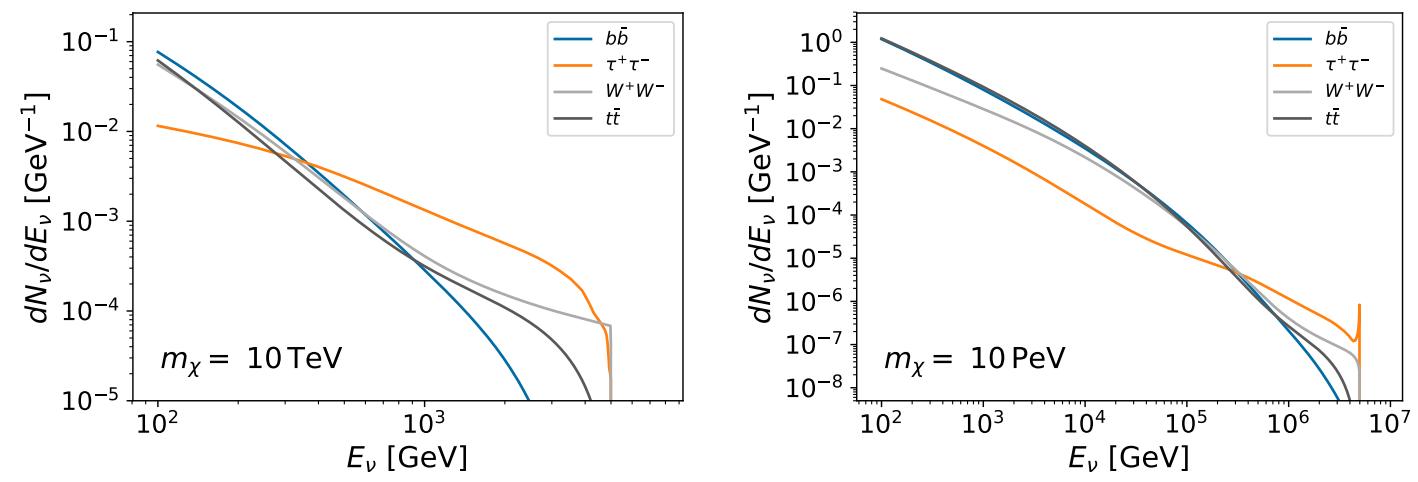

Figure 1: Differential energy spectra of $v_{\mu}$ (and $\bar{v}_{\mu}$ ) expected at the Earth for four different dark matter decay channels and two different dark matter masses. 


\begin{tabular}{|c|c|c|c|c|c|}
\hline Source & Type & $\mathrm{RA}\left[^{\circ}\right]$ & $\operatorname{Dec}\left[^{\circ}\right]$ & $\theta_{\max }\left[^{\circ}\right]$ & $D_{\max }\left[\mathrm{GeV} / \mathrm{cm}^{2}\right]$ \\
\hline \hline Virgo & galaxy cluster & 186.63 & 12.72 & 6.11 & $2.54 \times 10^{20}$ \\
Coma & galaxy cluster & 194.95 & 27.94 & 1.30 & $1.49 \times 10^{19}$ \\
Perseus & galaxy cluster & 49.94 & 41.51 & 1.35 & $1.44 \times 10^{19}$ \\
\hline Andromeda & galaxy & 10.68 & 41.27 & 8.00 & $1.70 \times 10^{20}$ \\
\hline Draco & dwarf galaxy & 260.05 & 57.92 & 1.30 & $1.54 \times 10^{19}$ \\
Leo I & dwarf galaxy & 152.12 & 12.3 & 0.45 & $1.19 \times 10^{18}$ \\
Ursa Minor & dwarf galaxy & 227.28 & 67.23 & 1.37 & $1.41 \times 10^{18}$ \\
Boötes I & dwarf galaxy & 210.03 & 14.5 & 0.47 & $1.46 \times 10^{18}$ \\
Coma Berenices & dwarf galaxy & 186.74 & 23.9 & 0.31 & $1.53 \times 10^{18}$ \\
Segue 1 & dwarf galaxy & 151.77 & 16.08 & 0.35 & $2.05 \times 10^{18}$ \\
Ursa Major II & dwarf galaxy & 132.87 & 63.13 & 0.53 & $5.23 \times 10^{18}$ \\
\hline
\end{tabular}

Table 1: Sources of neutrinos selected for this analysis. $\theta_{\max }$ is the angular radius of the dark matter halo of the source at which the D-factor, the integral factor in Eq. 1, reaches its maximum $\left(D_{\max }\right)$.

can be used to estimate the distribution of background events. This is done by replacing the right ascension of each event by a random value between 0 and $2 \pi$.

\section{Data Sample and Statistical Methods}

For this work, we use a subset of the IceCube data sample established to search for point-like sources [28]. The sample contains track-like events from both the southern and northern sky. The data are collected between 2008 and 2018 of which we use the last 6 years of data. The data of the subset are taken with the full 86-strings detector configuration, for which an updated event selection is applied compared to previous years. With this track-like event sample, the detector has a median angular resolution smaller than $1^{\circ}$, for neutrino energies of $\sim \mathrm{TeV}$ or above.

To determine whether there is a significant excess in data, we perform a hypothesis test using

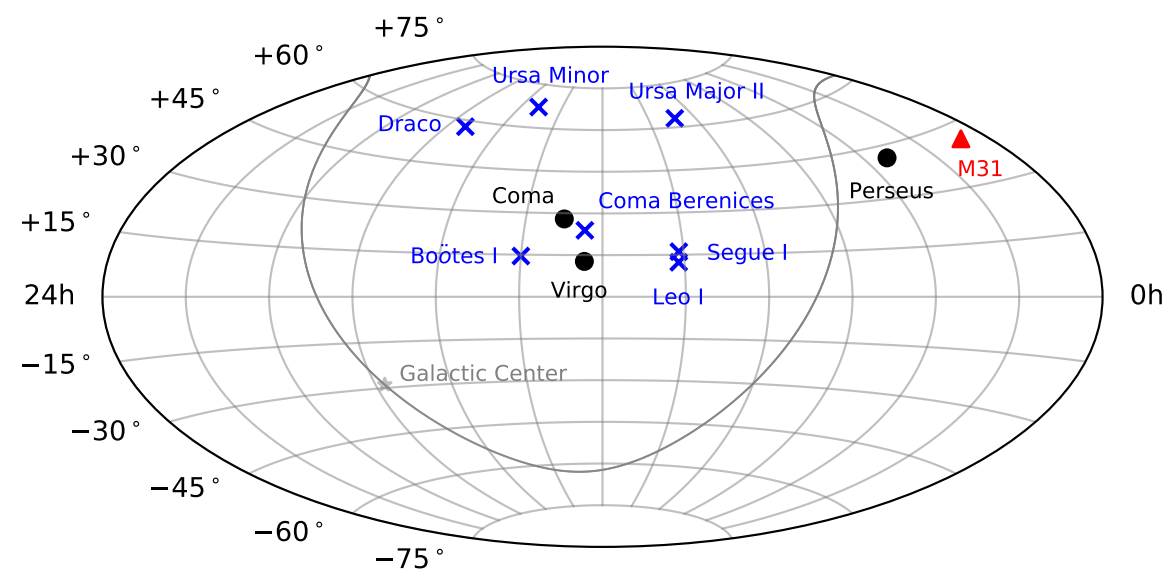

Figure 2: Locations of the sources in equatorial coordinates. The circles denote the location of the galaxy clusters. The crosses are for the dwarf galaxies, and the triangle is for the Andromeda galaxy (M31). For references, the locations of the Galactic Center (star) and the Galactic Plane (line) are also shown. 
a likelihood ratio test statistic defined as below:

$$
\lambda=-2 \log \frac{\mathcal{L}\left(n_{s}=0\right)}{\mathcal{L}\left(\hat{n}_{s}\right)},
$$

where $n_{s}$ is the expected number of signal events, and $\hat{n}_{s}$ is the best-fit value of the parameter. The likelihood function is defined as the following:

$$
\mathcal{L}\left(n_{s}\right)=\prod_{i=1}^{N}\left[\frac{n_{s}}{N} S\left(\alpha_{i}, \delta_{i}, \sigma_{i}, E_{i} \mid n_{s}\right)+\left(1-\frac{n_{s}}{N}\right) B\left(\alpha_{i}, \delta_{i}, \sigma_{i}, E_{i} \mid n_{s}\right)\right],
$$

where $i$ is the event index, and $N$ is the total number of observed events. $\alpha$ and $\delta$ are the reconstructed right ascension and declination, respectively. $\sigma$ is the angular uncertainty, and $E$ is the reconstructed neutrino energy.

The sensitivity is defined, at $90 \%$ confidence level (CL), as the minimum number of signal events required to have a Type I error rate smaller than 0.5 and Type II error rate of 0.1 . From this, we can deduce the sensitivity on the dark matter decay lifetime using the following equation:

$$
n_{s}=T_{\mathrm{live}} \int_{0}^{\Delta \Omega} d \Omega \int_{E_{\min }}^{E_{\max }} d E_{v} A_{\mathrm{eff}}\left(\hat{n}, E_{v}\right) \frac{d \Phi_{v}}{d \Omega d E_{v}}\left(\hat{n}, E_{v}\right),
$$

where $T_{\text {live }}$ is the detector livetime, $A_{\text {eff }}$ is the effective area of the detector, and $E_{\min }, E_{\max }$ are the minimum, maximum energies of the expected neutrinos, respectively.

\section{Sensitivities}

In this section, we present the $90 \% \mathrm{CL}$ sensitivities obtained for this analysis. The sensitivities computed for dark matter decaying into $b \bar{b}$ can be seen in Figure 3. The left panel shows the sensitivities for the galaxy clusters and the Andromeda galaxy. The sensitivities for the Virgo cluster are better than those for the Coma cluster and Perseus cluster. This is mainly due to the fact that the D-factor for the Virgo cluster is much larger than those for the other two clusters. Stacking the three clusters does not improve the sensitivities, compared to using only the Virgo cluster. The sensitivities for the Andromeda galaxy are comparable to those for the Virgo cluster. In the right panel of Figure 3, we present the sensitivities for the seven dwarf galaxies that we selected. The sensitivities for the Draco galaxy and the Ursa Major II galaxy are better than those for the other dwarf galaxies. This can be explained by the relatively large D-factors for the two galaxies, similar to the case of the galaxy clusters. The sensitivities are improved by stacking all of the dwarf galaxies, but they are still not comparable to the sensitivities for the Virgo cluster and the Andromeda galaxy.

Figure 4 shows the sensitivities for the $\tau^{+} \tau^{-}$channel. It can seen that for this channel the Virgo cluster and the Andromeda galaxy are the most promising sources. Stacking the dwarf galaxies leads to slightly better sensitivities than those for the best dwarf galaxy. The sensitivity curves for Perseus, Andromeda, Draco, Ursa Major II, and Ursa Minor have onsets of decrease. This is due to the opacity of the Earth to neutrinos. The opacity increases with increasing neutrino energy and increasing source declination. Thus, the Earth is more opaque to the neutrinos from these sources than the others. Furthermore, the higher the dark matter mass is, the larger is the opacity for these 

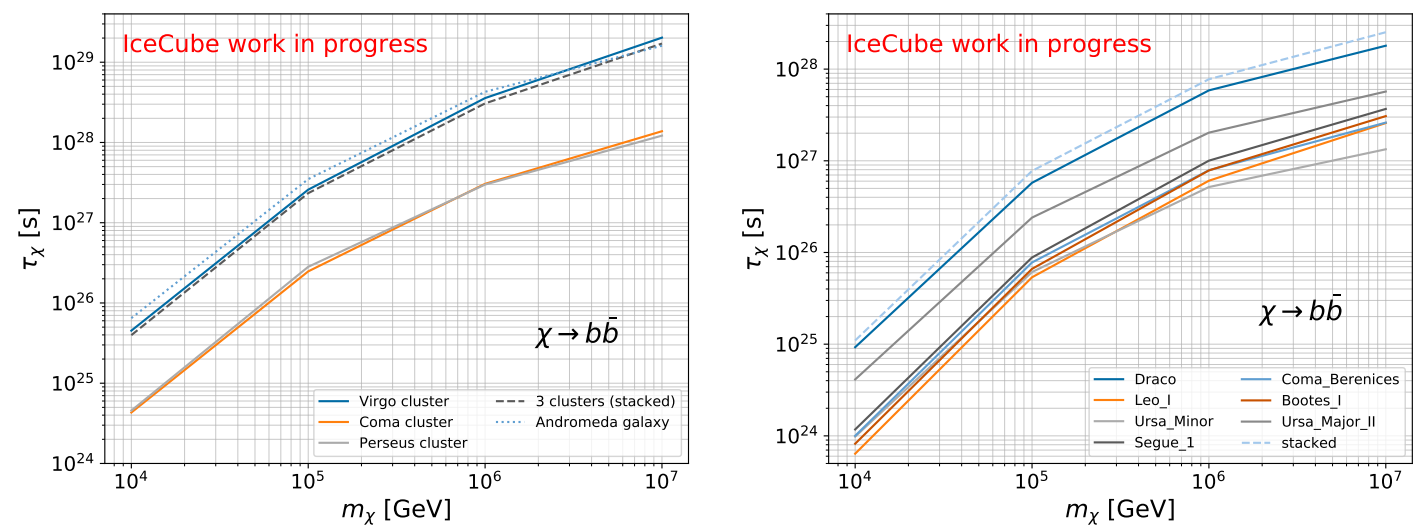

Figure 3: $90 \%$ CL sensitivities on dark matter lifetime for the $b \bar{b}$ channel. Left: The sensitivities for the three galaxy clusters. The solid lines are for the individual galaxy clusters, and the dashed line is for the case of stacking the three clusters. The dotted line is for the Andromeda galaxy. Right: The sensitivities for the seven dwarf galaxies. The solid lines are for the individual dwarf galaxies and the dashed line is for the case of stacking all of the dwarf galaxies.

sources. Therefore, the sensitivities for the sources do not keep increasing with increasing dark matter mass. This effect is less noticeable when dark matter decays into $b \bar{b}$, as the neutrino spectra for this channel are soft.

We compare the sensitivities of this analysis to the observed limits from other experiments in Figure 5. The dashed line represents the sensitivities of the presented analysis for the $b \bar{b}$ channel and the Andromeda galaxy. The solid lines are the limits from different experiments [16, 29-32], for the same dark matter decay channel. It can be seen that the sensitivities calculated for this analysis are competitive with other experiments.
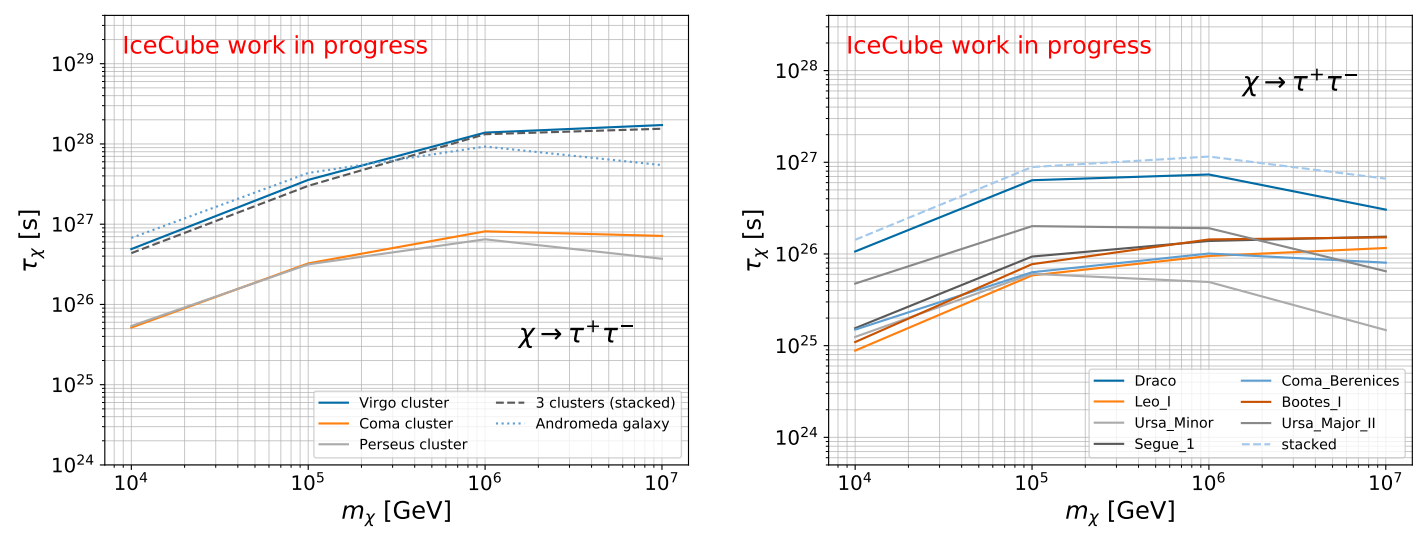

Figure 4: $90 \% \mathrm{CL}$ sensitivities on dark matter lifetime for the $\tau^{+} \tau^{-}$channel. 


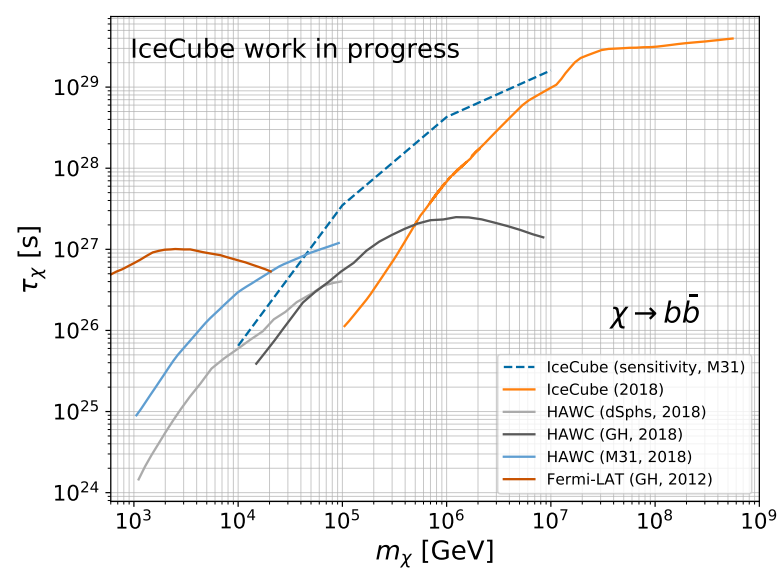

Figure 5: Comparison of the sensitivities of the analysis to the observed limits from other experiments [16, 29-32]. The dashed line represents the 90\% CL sensitivities of the analysis for the $b \bar{b}$ channel and the Andromeda galaxy. Other lines are the limits from different experiments calculated assuming the same dark matter decay channel. The confidence levels associated with the IceCube limits, HAWC limits, and the Fermi-LAT limits are $90 \%, 95 \%$, and $3 \sigma$, respectively.

\section{Conclusions and Outlook}

We calculated the sensitivities, at $90 \% \mathrm{CL}$, for the $b \bar{b}, \tau^{+} \tau^{-}$channels for dark matter masses between $10 \mathrm{TeV}$ and $10 \mathrm{PeV}$. For the Virgo cluster and the Andromeda galaxy, the sensitivities are competitive with other leading experiments. We also tested if the sensitivities could be improved by stacking the sources. For the $b \bar{b}$ and $\tau^{+} \tau^{-}$channels, stacking the galaxy clusters did not improve the sensitivities, compared to using only the Virgo cluster. Furthermore, the sensitivities for the Virgo cluster and the Andromeda galaxy were significantly better than those obtained by stacking the dwarf galaxies. We plan to study the sensitivities for other dark matter decay channels than $b \bar{b}$ and $\tau^{+} \tau^{-}$. Galaxy clusters and dwarf galaxies in the southern sky could be included in the list of targets, and the benefit of stacking could be evaluated again.

\section{References}

[1] G. Steigman, B. Dasgupta, and J. F. Beacom Phys. Rev. D 86 (2012) 023506.

[2] K. Griest and M. Kamionkowski Phys. Rev. Lett. 64 (1990) 615.

[3] K. Murase and J. F. Beacom JCAP 10 (2012) 043.

[4] C. Rott, K. Kohri, and S. C. Park Phys. Rev. D 92 no. 2, (2015) 023529.

[5] IceCube Collaboration, M. G. Aartsen et al. Eur. Phys. J. C 77 no. 3, (2017) 146. [Erratum: Eur.Phys.J.C 79, 214 (2019)].

[6] IceCube Collaboration, M. G. Aartsen et al. Eur. Phys. J. C 77 no. 2, (2017) 82.

[7] ANTARES Collaboration, S. Adrián-Martínez EPJ Web Conf. 121 (2016) 06004. 
[8] IceCube Collaboration, M. G. Aartsen et al. JINST 12 no. 03, (2017) P03012.

[9] IceCube Collaboration, M. G. Aartsen et al. Science 342 (2013) 1242856.

[10] IceCube Collaboration, M. G. Aartsen et al. Phys. Rev. Lett. 113 (2014) 101101.

[11] IceCube Collaboration, M. G. Aartsen et al. Phys. Rev. Lett. 115 no. 8, (2015) 081102.

[12] A. Esmaili, S. K. Kang, and P. D. Serpico JCAP 12 (2014) 054.

[13] S. M. Boucenna, M. Chianese, G. Mangano, G. Miele, S. Morisi, O. Pisanti, and E. Vitagliano JCAP 12 (2015) 055.

[14] M. Chianese, G. Miele, and S. Morisi Phys. Lett. B 773 (2017) 591-595.

[15] A. Bhattacharya, A. Esmaili, S. Palomares-Ruiz, and I. Sarcevic JCAP 05 (2019) 051.

[16] IceCube Collaboration, M. G. Aartsen et al. Eur. Phys. J. C 78 no. 10, (2018) 831.

[17] IceCube Collaboration, C. A. Argüelles and H. Dujmovic PoS ICRC2019 (2020) 839.

[18] Q. Liu, J. Lazar, C. A. Argüelles, and A. Kheirandish JCAP 10 (2020) 043.

[19] A. Gazizov and M. P. Kowalski Comput. Phys. Commun. 172 (2005) 203-213.

[20] A. Geringer-Sameth, S. M. Koushiappas, and M. Walker Astrophys. J. 801 no. 2, (2015) 74.

[21] H. Zhao Mon. Not. Roy. Astron. Soc. 278 (1996) 488-496.

[22] M. A. Sanchez-Conde, M. Cannoni, F. Zandanel, M. E. Gomez, and F. Prada JCAP 12 (2011) 011.

[23] J. F. Navarro, C. S. Frenk, and S. D. M. White Astrophys. J. 490 (1997) 493-508.

[24] A. Tamm, E. Tempel, P. Tenjes, O. Tihhonova, and T. Tuvikene Astron. Astrophys. 546 (2012) A4.

[25] A. W. Graham, D. Merritt, B. Moore, J. Diemand, and B. Terzic Astron. J. 132 (2006) 2685-2700.

[26] V. Bonnivard, M. Hütten, E. Nezri, A. Charbonnier, C. Combet, and D. Maurin Comput. Phys. Commun. 200 (2016) 336-349.

[27] M. Hütten, C. Combet, and D. Maurin Comput. Phys. Commun. 235 (2019) 336-345.

[28] IceCube Collaboration, M. G. Aartsen et al. Phys. Rev. Lett. 124 no. 5, (2020) 051103.

[29] HAWC Collaboration, A. Albert et al. JCAP 06 (2018) 043. [Erratum: JCAP 04, E01 (2019)].

[30] HAWC Collaboration, A. Albert et al. Astrophys. J. 853 no. 2, (2018) 154.

[31] HAWC Collaboration, A. U. Abeysekara et al. JCAP 02 (2018) 049.

[32] Fermi-LAT Collaboration, M. Ackermann et al. Astrophys. J. 761 (2012) 91. 


\section{Full Authors List: Collaboration}

R. Abbasi ${ }^{17}$, M. Ackermann ${ }^{59}$, J. Adams ${ }^{18}$, J. A. Aguilar ${ }^{12}$, M. Ahlers ${ }^{22}$, M. Ahrens ${ }^{50}$, C. Alispach ${ }^{28}$, A. A. Alves Jr. ${ }^{31}$, N. M. $\mathrm{Amin}^{42}$, R. An ${ }^{14}$, K. Andeen ${ }^{40}$, T. Anderson ${ }^{56}$, G. Anton ${ }^{26}$, C. Argüelles ${ }^{14}$, Y. Ashida ${ }^{38}$, S. Axani ${ }^{15}$, X. Bai ${ }^{46}$, A. Balagopal V. ${ }^{38}$, A. Barbano ${ }^{28}$, S. W. Barwick ${ }^{30}$, B. Bastian ${ }^{59}$, V. Basu ${ }^{38}$, S. Baur ${ }^{12}$, R. Bay ${ }^{8}$, J. J. Beatty ${ }^{20,21}$, K.-H. Becker ${ }^{58}$, J. Becker Tjus ${ }^{11}$, C. Bellenghi $^{27}$, S. BenZvi ${ }^{48}$, D. Berley ${ }^{19}$, E. Bernardini ${ }^{59,60}$, D. Z. Besson ${ }^{34,61}$, G. Binder ${ }^{8,9}$, D. Bindig ${ }^{58}$, E. Blaufuss ${ }^{19}$, S. Blot ${ }^{59}$, M. Boddenberg ${ }^{1}$, F. Bontempo ${ }^{31}$, J. Borowka ${ }^{1}$, S. Böser ${ }^{39}$, O. Botner ${ }^{57}$, J. Böttcher ${ }^{1}$, E. Bourbeau ${ }^{22}$, F. Bradascio ${ }^{59}$, J. Braun ${ }^{38}$, S. Bron $^{28}$, J. Brostean-Kaiser ${ }^{59}$, S. Browne ${ }^{32}$, A. Burgman ${ }^{57}$, R. T. Burley ${ }^{2}$, R. S. Busse ${ }^{41}$, M. A. Campana ${ }^{45}$, E. G. Carnie-Bronca ${ }^{2}$, C. Chen ${ }^{6}$, D. Chirkin ${ }^{38}$, K. Choi ${ }^{52}$, B. A. Clark ${ }^{24}$, K. Clark ${ }^{33}$, L. Classen ${ }^{41}$, A. Colemann ${ }^{42}$, G. H. Collin ${ }^{15}$, J. M. Conrad ${ }^{15}$, P. Coppin $^{13}$, P. Correa ${ }^{13}$, D. F. Cowen ${ }^{55,56}$, R. Cross $^{48}$, C. Dappen ${ }^{1}$, P. Dave ${ }^{6}$, C. De Clercq ${ }^{13}$, J. J. DeLaunay ${ }^{56}$, H. Dembinski ${ }^{42}$, K. Deoskar $^{50}$, S. De Ridder ${ }^{29}$, A. Desai ${ }^{38}$, P. Desiati ${ }^{38}$, K. D. de Vries ${ }^{13}$, G. de Wasseige ${ }^{13}$, M. de With ${ }^{10}$, T. DeYoung ${ }^{24}$, S. Dharani ${ }^{1}$, A. Diaz ${ }^{15}$, J. C. Díaz-Vélez ${ }^{38}$, M. Dittmer ${ }^{41}$, H. Dujmovic ${ }^{31}$, M. Dunkman ${ }^{56}$, M. A. DuVernois ${ }^{38}$, E. Dvorak ${ }^{46}$, T. Ehrhardt ${ }^{39}$, P. Eller $^{27}$, R. Engel ${ }^{31,32}$, H. Erpenbeck ${ }^{1}$, J. Evans ${ }^{19}$, P. A. Evenson ${ }^{42}$, K. L. Fan ${ }^{19}$, A. R. Fazely ${ }^{7}$, S. Fiedlschuster ${ }^{26}$, A. T. Fienberg ${ }^{56}$, K. Filimonov ${ }^{8}$, C. Finley ${ }^{50}$, L. Fischer ${ }^{59}$, D. Fox ${ }^{55}$, A. Franckowiak ${ }^{11,59}$, E. Friedman ${ }^{19}$, A. Fritz ${ }^{39}$, P. Fürst ${ }^{1}$, T. K. Gaisser ${ }^{42}$, J. Gallagher $^{37}$, E. Ganster ${ }^{1}$, A. Garcia ${ }^{14}$, S. Garrappa ${ }^{59}$, L. Gerhardt ${ }^{9}$, A. Ghadimi ${ }^{54}$, C. Glaser ${ }^{57}$, T. Glauch ${ }^{27}$, T. Glüsenkamp ${ }^{26}$, A. Goldschmidt $^{9}$, J. G. Gonzalez ${ }^{42}$, S. Goswami ${ }^{54}$, D. Grant ${ }^{24}$, T. Grégoire ${ }^{56}$, S. Griswold ${ }^{48}$, M. Gündüz ${ }^{11}$, C. Günther ${ }^{1}$, C. Haack ${ }^{27}$,

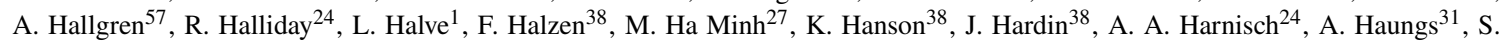
Hauser $^{1}$, D. Hebecker ${ }^{10}$, K. Helbing ${ }^{58}$, F. Henningsen ${ }^{27}$, E. C. Hettinger ${ }^{24}$, S. Hickford ${ }^{58}$, J. Hignight ${ }^{25}$, C. Hill ${ }^{16}$, G. C. Hill ${ }^{2}$, K. D.

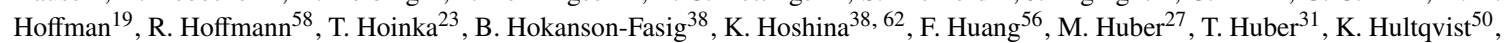
M. Hünnefeld ${ }^{23}$, R. Hussain ${ }^{38}$, S. In ${ }^{52}$, N. Iovine ${ }^{12}$, A. Ishihara ${ }^{16}$, M. Jansson ${ }^{50}$, G. S. Japaridze ${ }^{5}$, M. Jeong ${ }^{52}$, B. J. P. Jones ${ }^{4}$, D. Kang ${ }^{31}$, W. Kang ${ }^{52}$, X. Kang ${ }^{45}$, A. Kappes ${ }^{41}$, D. Kappesser ${ }^{39}$, T. Karg ${ }^{59}$, M. Karl ${ }^{27}$, A. Karle ${ }^{38}$, U. Katz ${ }^{26}$, M. Kauer ${ }^{38}$, M. Kellermann ${ }^{1}$, J. L. Kelley $^{38}$, A. Kheirandish ${ }^{56}$, K. Kin ${ }^{16}$, T. Kintscher ${ }^{59}$, J. Kiryluk ${ }^{51}$, S. R. Klein ${ }^{8,9}$, R. Koirala ${ }^{42}$, H. Kolanoski ${ }^{10}$, T. Kontrimas ${ }^{27}$, L. Köpke $^{39}$, C. Kopper ${ }^{24}$, S. Kopper ${ }^{54}$, D. J. Koskinen ${ }^{22}$, P. Koundal ${ }^{31}$, M. Kovacevich ${ }^{45}$, M. Kowalski ${ }^{10,59}$, T. Kozynets ${ }^{22}$, E. Kun ${ }^{11}$, N. Kurahashi ${ }^{45}$, N. Lad ${ }^{59}$, C. Lagunas Gualda ${ }^{59}$, J. L. Lanfranchi ${ }^{56}$, M. J. Larson ${ }^{19}$, F. Lauber ${ }^{58}$, J. P. Lazar ${ }^{14,38}$, J. W. Lee ${ }^{52}$, K. Leonard $^{38}$, A. Leszczyńska ${ }^{32}$, Y. Li ${ }^{56}$, M. Lincetto ${ }^{11}$, Q. R. Liu ${ }^{38}$, M. Liubarska ${ }^{25}$, E. Lohfink ${ }^{39}$, C. J. Lozano Mariscal ${ }^{41}$, L. Lu ${ }^{38}$,



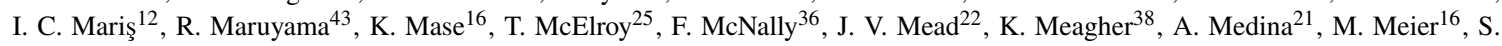
Meighen-Berger $^{27}$, J. Micallef ${ }^{24}$, D. Mockler ${ }^{12}$, T. Montaruli ${ }^{28}$, R. W. Moore ${ }^{25}$, R. Morse ${ }^{38}$, M. Moulai ${ }^{15}$, R. Naab ${ }^{59}$, R. Nagai ${ }^{16}$, U. Naumann $^{58}$, J. Necker ${ }^{59}$, L. V. Nguyễn ${ }^{24}$, H. Niederhausen ${ }^{27}$, M. U. Nisa ${ }^{24}$, S. C. Nowicki ${ }^{24}$, D. R. Nygren ${ }^{9}$, A. Obertacke Pollmann ${ }^{58}$, M. Oehler ${ }^{31}$, A. Olivas ${ }^{19}$, E. O'Sullivan ${ }^{57}$, H. Pandya ${ }^{42}$, D. V. Pankova ${ }^{56}$, N. Park ${ }^{33}$, G. K. Parker ${ }^{4}$, E. N. Paudel ${ }^{42}$, L. Paul ${ }^{40}$, C. Pérez de los $\operatorname{Heros}^{57}$, L. Peters ${ }^{1}$, J. Peterson ${ }^{38}$, S. Philippen ${ }^{1}$, D. Pieloth ${ }^{23}$, S. Pieper ${ }^{58}$, M. Pittermann ${ }^{32}$, A. Pizzuto ${ }^{38}$, M. Plum ${ }^{40}$, Y. Popovych $^{39}$, A. Porcelli ${ }^{29}$, M. Prado Rodriguez ${ }^{38}$, P. B. Price ${ }^{8}$, B. Pries ${ }^{24}$, G. T. Przybylski ${ }^{9}$, C. Raab ${ }^{12}$, A. Raissi ${ }^{18}$, M. Rameez ${ }^{22}$, K. $^{2}$. Rawlins $^{3}$, I. C. Rea ${ }^{27}$, A. Rehman ${ }^{42}$, P. Reichherzer ${ }^{11}$, R. Reimann ${ }^{1}$, G. Renzi ${ }^{12}$, E. Resconi ${ }^{27}$, S. Reusch ${ }^{59}$, W. Rhode ${ }^{23}$, M. Richman $^{45}$, B. Riedel ${ }^{38}$, E. J. Roberts ${ }^{2}$, S. Robertson ${ }^{8,9}$, G. Roellinghoff ${ }^{52}$, M. Rongen ${ }^{39}$, C. Rott ${ }^{49}, 52$, T. Ruhe ${ }^{23}$, D. Ryckbosch ${ }^{29}$, D. Rysewyk $\mathrm{Cantu}^{24}$, I. Safa ${ }^{14,38}$, J. Saffer ${ }^{32}$, S. E. Sanchez Herrera ${ }^{24}$, A. Sandrock ${ }^{23}$, J. Sandroos ${ }^{39}$, M. Santander ${ }^{54}$, S. Sarkar ${ }^{44}$, S. Sarkar ${ }^{25}$, K. Satalecka $^{59}$, M. Scharf ${ }^{1}$, M. Schaufel ${ }^{1}$, H. Schieler ${ }^{31}$, S. Schindler ${ }^{26}$, P. Schlunder ${ }^{23}$, T. Schmidt ${ }^{19}$, A. Schneider ${ }^{38}$, J. Schneider ${ }^{26}$, F. G. Schröder ${ }^{31,42}$, L. Schumacher ${ }^{27}$, G. Schwefer ${ }^{1}$, S. Sclafani ${ }^{45}$, D. Seckel ${ }^{42}$, S. Seunarine ${ }^{47}$, A. Sharma ${ }^{57}$, S. Shefali ${ }^{32}$, M. Silva $^{38}$, B. Skrzypek ${ }^{14}$, B. Smithers ${ }^{4}$, R. Snihur ${ }^{38}$, J. Soedingrekso ${ }^{23}$, D. $\operatorname{Soldin}^{42}$, C. Spannfellner ${ }^{27}$, G. M. Spiczak ${ }^{47}$, C. Spiering ${ }^{59,61}$, J. Stachurska $^{59}$, M. Stamatikos ${ }^{21}$, T. Stanev ${ }^{42}$, R. Stein ${ }^{59}$, J. Stettner ${ }^{1}$, A. Steuer ${ }^{39}$, T. Stezelberger ${ }^{9}$, T. Stürwald ${ }^{58}$, T. Stuttard ${ }^{22}$, G. W. Sullivan $^{19}$, I. Taboada ${ }^{6}$, F. Tenholt ${ }^{11}$, S. Ter-Antonyan ${ }^{7}$, S. Tilav ${ }^{42}$, F. Tischbein ${ }^{1}$, K. Tollefson $^{24}$, L. Tomankova ${ }^{11}$, C. Tönnis ${ }^{53}$, S.

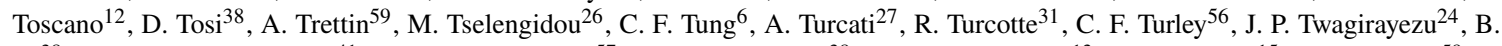
$\mathrm{Ty}^{38}$, M. A. Unland Elorrieta ${ }^{41}$, N. Valtonen-Mattila ${ }^{57}$, J. Vandenbroucke ${ }^{38}$, N. van Eijndhoven ${ }^{13}$, D. Vannerom ${ }^{15}$, J. van Santen ${ }^{59}, \mathrm{~S}$. Verpoest $^{29}$, M. Vraeghe ${ }^{29}$, C. Walck ${ }^{50}$, T. B. Watson ${ }^{4}$, C. Weaver ${ }^{24}$, P. Weigel ${ }^{15}$, A. Weindl ${ }^{31}$, M. J. Weiss ${ }^{56}$, J. Weldert ${ }^{39}$, C. Wendt $^{38}$, J. Werthebach ${ }^{23}$, M. Weyrauch ${ }^{32}$, N. Whitehorn ${ }^{24,35}$, C. H. Wiebusch ${ }^{1}$, D. R. Williams ${ }^{54}$, M. Wolf ${ }^{27}$, K. Woschnagg ${ }^{8}$, G. Wrede ${ }^{26}$, J. Wulff ${ }^{11}$, X. W. Xu $u^{7}$, Y. Xu ${ }^{51}$, J. P. Yanez ${ }^{25}$, S. Yoshida ${ }^{16}$, S. Yu ${ }^{24}$, T. Yuan ${ }^{38}$, Z. Zhang ${ }^{51}$

${ }^{1}$ III. Physikalisches Institut, RWTH Aachen University, D-52056 Aachen, Germany

2 Department of Physics, University of Adelaide, Adelaide, 5005, Australia

${ }^{3}$ Dept. of Physics and Astronomy, University of Alaska Anchorage, 3211 Providence Dr., Anchorage, AK 99508, USA

${ }^{4}$ Dept. of Physics, University of Texas at Arlington, 502 Yates St., Science Hall Rm 108, Box 19059, Arlington, TX 76019, USA

5 CTSPS, Clark-Atlanta University, Atlanta, GA 30314, USA

${ }^{6}$ School of Physics and Center for Relativistic Astrophysics, Georgia Institute of Technology, Atlanta, GA 30332, USA

${ }^{7}$ Dept. of Physics, Southern University, Baton Rouge, LA 70813, USA

${ }^{8}$ Dept. of Physics, University of California, Berkeley, CA 94720, USA

${ }^{9}$ Lawrence Berkeley National Laboratory, Berkeley, CA 94720, USA

${ }^{10}$ Institut für Physik, Humboldt-Universität zu Berlin, D-12489 Berlin, Germany

${ }^{11}$ Fakultät für Physik \& Astronomie, Ruhr-Universität Bochum, D-44780 Bochum, Germany

12 Université Libre de Bruxelles, Science Faculty CP230, B-1050 Brussels, Belgium

13 Vrije Universiteit Brussel (VUB), Dienst ELEM, B-1050 Brussels, Belgium

14 Department of Physics and Laboratory for Particle Physics and Cosmology, Harvard University, Cambridge, MA 02138, USA

15 Dept. of Physics, Massachusetts Institute of Technology, Cambridge, MA 02139, USA 
${ }^{16}$ Dept. of Physics and Institute for Global Prominent Research, Chiba University, Chiba 263-8522, Japan

${ }^{17}$ Department of Physics, Loyola University Chicago, Chicago, IL 60660, USA

${ }^{18}$ Dept. of Physics and Astronomy, University of Canterbury, Private Bag 4800, Christchurch, New Zealand

${ }^{19}$ Dept. of Physics, University of Maryland, College Park, MD 20742, USA

${ }^{20}$ Dept. of Astronomy, Ohio State University, Columbus, OH 43210, USA

${ }^{21}$ Dept. of Physics and Center for Cosmology and Astro-Particle Physics, Ohio State University, Columbus, OH 43210, USA

${ }^{22}$ Niels Bohr Institute, University of Copenhagen, DK-2100 Copenhagen, Denmark

${ }^{23}$ Dept. of Physics, TU Dortmund University, D-44221 Dortmund, Germany

24 Dept. of Physics and Astronomy, Michigan State University, East Lansing, MI 48824, USA

${ }^{25}$ Dept. of Physics, University of Alberta, Edmonton, Alberta, Canada T6G 2E1

${ }^{26}$ Erlangen Centre for Astroparticle Physics, Friedrich-Alexander-Universität Erlangen-Nürnberg, D-91058 Erlangen, Germany

${ }^{27}$ Physik-department, Technische Universität München, D-85748 Garching, Germany

${ }^{28}$ Département de physique nucléaire et corpusculaire, Université de Genève, CH-1211 Genève, Switzerland

${ }^{29}$ Dept. of Physics and Astronomy, University of Gent, B-9000 Gent, Belgium

${ }^{30}$ Dept. of Physics and Astronomy, University of California, Irvine, CA 92697, USA

${ }^{31}$ Karlsruhe Institute of Technology, Institute for Astroparticle Physics, D-76021 Karlsruhe, Germany

${ }^{32}$ Karlsruhe Institute of Technology, Institute of Experimental Particle Physics, D-76021 Karlsruhe, Germany

${ }^{3}$ Dept. of Physics, Engineering Physics, and Astronomy, Queen's University, Kingston, ON K7L 3N6, Canada

${ }^{34}$ Dept. of Physics and Astronomy, University of Kansas, Lawrence, KS 66045, USA

${ }^{35}$ Department of Physics and Astronomy, UCLA, Los Angeles, CA 90095, USA

${ }^{36}$ Department of Physics, Mercer University, Macon, GA 31207-0001, USA

${ }^{37}$ Dept. of Astronomy, University of Wisconsin-Madison, Madison, WI 53706, USA

${ }^{38}$ Dept. of Physics and Wisconsin IceCube Particle Astrophysics Center, University of Wisconsin-Madison, Madison, WI 53706, USA

${ }^{39}$ Institute of Physics, University of Mainz, Staudinger Weg 7, D-55099 Mainz, Germany

${ }^{40}$ Department of Physics, Marquette University, Milwaukee, WI, 53201, USA

${ }^{41}$ Institut für Kernphysik, Westfälische Wilhelms-Universität Münster, D-48149 Münster, Germany

${ }^{42}$ Bartol Research Institute and Dept. of Physics and Astronomy, University of Delaware, Newark, DE 19716, USA

${ }^{43}$ Dept. of Physics, Yale University, New Haven, CT 06520, USA

${ }^{44}$ Dept. of Physics, University of Oxford, Parks Road, Oxford OX1 3PU, UK

${ }^{45}$ Dept. of Physics, Drexel University, 3141 Chestnut Street, Philadelphia, PA 19104, USA

${ }^{46}$ Physics Department, South Dakota School of Mines and Technology, Rapid City, SD 57701, USA

${ }^{47}$ Dept. of Physics, University of Wisconsin, River Falls, WI 54022, USA

${ }^{48}$ Dept. of Physics and Astronomy, University of Rochester, Rochester, NY 14627, USA

${ }^{49}$ Department of Physics and Astronomy, University of Utah, Salt Lake City, UT 84112, USA

${ }^{50}$ Oskar Klein Centre and Dept. of Physics, Stockholm University, SE-10691 Stockholm, Sweden

${ }^{51}$ Dept. of Physics and Astronomy, Stony Brook University, Stony Brook, NY 11794-3800, USA

52 Dept. of Physics, Sungkyunkwan University, Suwon 16419, Korea

${ }^{53}$ Institute of Basic Science, Sungkyunkwan University, Suwon 16419, Korea

${ }^{54}$ Dept. of Physics and Astronomy, University of Alabama, Tuscaloosa, AL 35487, USA

${ }^{55}$ Dept. of Astronomy and Astrophysics, Pennsylvania State University, University Park, PA 16802, USA

${ }^{56}$ Dept. of Physics, Pennsylvania State University, University Park, PA 16802, USA

57 Dept. of Physics and Astronomy, Uppsala University, Box 516, S-75120 Uppsala, Sweden

${ }^{58}$ Dept. of Physics, University of Wuppertal, D-42119 Wuppertal, Germany

${ }^{59}$ DESY, D-15738 Zeuthen, Germany

${ }^{60}$ Università di Padova, I-35131 Padova, Italy

${ }^{61}$ National Research Nuclear University, Moscow Engineering Physics Institute (MEPhI), Moscow 115409, Russia

${ }^{62}$ Earthquake Research Institute, University of Tokyo, Bunkyo, Tokyo 113-0032, Japan

\section{Acknowledgements}

USA - U.S. National Science Foundation-Office of Polar Programs, U.S. National Science Foundation-Physics Division, U.S. National Science Foundation-EPSCoR, Wisconsin Alumni Research Foundation, Center for High Throughput Computing (CHTC) at the University of Wisconsin-Madison, Open Science Grid (OSG), Extreme Science and Engineering Discovery Environment (XSEDE), Frontera computing project at the Texas Advanced Computing Center, U.S. Department of Energy-National Energy Research Scientific Computing Center, Particle astrophysics research computing center at the University of Maryland, Institute for Cyber-Enabled Research at Michigan State University, and Astroparticle physics computational facility at Marquette University; Belgium - Funds for Scientific Research (FRS-FNRS and FWO), FWO Odysseus and Big Science programmes, and Belgian Federal Science Policy Office (Belspo); Germany - Bundesministerium für Bildung und Forschung (BMBF), Deutsche Forschungsgemeinschaft (DFG), Helmholtz Alliance for Astroparticle Physics (HAP), Initiative and Networking Fund of the Helmholtz Association, Deutsches Elektronen Synchrotron (DESY), and High Performance Computing cluster of the RWTH Aachen; Sweden - Swedish Research Council, Swedish Polar Research Secretariat, Swedish National Infrastructure for Computing (SNIC), and Knut and Alice Wallenberg Foundation; Australia - Australian 
Research Council; Canada - Natural Sciences and Engineering Research Council of Canada, Calcul Québec, Compute Ontario, Canada Foundation for Innovation, WestGrid, and Compute Canada; Denmark - Villum Fonden and Carlsberg Foundation; New Zealand Marsden Fund; Japan - Japan Society for Promotion of Science (JSPS) and Institute for Global Prominent Research (IGPR) of Chiba University; Korea - National Research Foundation of Korea (NRF); Switzerland - Swiss National Science Foundation (SNSF); United Kingdom - Department of Physics, University of Oxford. 